\title{
Open Source Geospatial Foundationopen source geospatial foundation
}

The Open Source Geospatial Foundation (OSGeo) is a U.S.-based not-for-profit organization that supports and promotes the use and collaborative development of open-s ource geospatial technologies and data around the world. OSGeo offers marketing, financial, organizational, and legal help for its projects. The mission includes advocacy and marketing and encourages the development of business models based on Open Source GIS (OSGIS). The foundation acts as an advisory body for OSGIS software projects as well as educational material and public geos patial data development projects. Communication is based on classical methods and newer Web 2.0 tools: Wikis, issue trackers, mailing lists, Web sites, Internet Relay Chat, and blogs.

\section{Hist ory}

OSGIS-related user and developer communities have existed since the early 1980s. They expanded significantly with the advent of Internet and electronic communication. Each relevant OSGIS project had its own community, in most cases only in virtual form and without formal structures. In response to the increasing need of collective support of OSGIS s oftware development efforts, the Open Source Geos patial Foundation was officially created in February 2006 as a not-for-profit organization.

\section{OSGeo Projects}

OSGIS-related software projects that want to become part of the foundation have to undergo an incubation phase to verify if the project conforms with OSGeo principles. Accepted projects demonstrate mature project management, with open and collaborative development and user communities. Increasingly, real-time code quality assessment systems are implemented. A review of the intellectual property of source code and documentation has been performed. OSGeo software projects include geos patial-programming libraries, desktop GIS, Web mapping/WebGIS, and metadata catalogs.

In addition to software dissemination, OSGeo aims at educational outreach. Part of the mission is to promote OSGIS training among universities and other educational institutions; OSGeo is preparing and providing training materials and teaching support released under free licenses.

Third, OSGeo promotes the use of open geospa-tial data formats and public access to state-collected geos patial data. In collaboration with other entities, licenses for public geos patial data are developed with adaptation to various legislations.

\section{OSGeo Governance}

OSGeo is a community-driven and distributed organization with a formal seat in the United States but legal components distributed in various countries. It consists of an elected board of directors, an executive director, elected charter members (eligible to vote for the board), and members-at-large. The members collaborate through various projects, committees, and local chapters, which repres ent OSGeo around the world in different countries and language groups. All projects are managed through individual project steering committees (PSCs) with PSC chairpers ons serving as foundation officers .

OSGeo annually presents the Sol Katz Award for Geospatial Free and Open Source Software (GFOSS) to individuals who have demonstrated leadership in the wider GFOSS community. Recipients of the award are recognized for their significant contribution in the geos patial realm to advance open-s ource ideals.

Since the early days of OSGIS communities, annual conferences at national and international levels were organized. In 2006, OSGeo merged several of their community events into the "Free and Open Source Software for Geoinformatics" (FOSS4G) conference series, which is hosted each year in a different country. In addition to the common conference scheme, these conferences include a 5-minute plenary, "Lightning Talks," and "code sprints," where project teams get together for valuable face-to-face planning and concentrated programming time.

Markus Neteler 


\section{Further Readings}

Hall, G. B. , ed. , \& Leahy, M. G. (Eds.). (2008). Open source approaches in spatial data handling (Advances in Geographic Information Science Series). New York: Springer.

Open Source Geospatial Foundation: www.osgeo.org

\section{Entry Cit ation:}

Neteler, Markus. "Open Source Geos patial Foundation." Encyclopedia of Geography. 2010. SAGE Publications. 2 Oct. 2010. <http://www.s age-ereference.com/geography/Article_n844.html>.

\section{(\$)SAGE}

(C) SAGE Publications, Inc. 